\title{
A Causality Algorithm for Magnetic Hysteresis of Interacting Systems
}

\author{
Jyh-Shinn Yang \\ Division of General Education, National Taiwan Ocean University, Keelung, TAIWAN, R. O. C. \\ Ching-Ray Chang and Ivo Klik \\ Department of Physics, National Taiwan University, Taipei, TAIWAN, R. O.C.
}

\begin{abstract}
A causality algorithm is introduced to keep track of all history of interacting magnetic systems since saturation. It reflects the true hysteresis of the interacting system with ambient temperature. Though not entirely self-consistent, it satisfies the requirement of causality and is physically more realistic. Because it contains the evolution due to the interactions, thus the coercivity indeed shifts linearly with the coupling strength.
\end{abstract}

\section{INTRODUCTION}

Magnetic hysteresis for an interacting system has been under extensive studies [1-4], however, a lot of phenomena still are not fully understood. Hysteresis, i.e., memory, effects are associated with the presence of local metastable energy minima in which, in the absence of the understanding of the energy surface of the interacting system, the studies of hysteresis remain indefinitely locked. A vectorial modelling of hysteresis is based on the fact that Stoner-Wohlfarth (S-W) uniform mode [5] is designed as an ensemble of single-domain, uniaxial magnetic particles. Given the system energy, there are two common approaches to determine the spin configuration. One method uses the local field [6] to sequentially iterate the magnetization of each particle and then determines spin configurations. An alternative approach is to solve the LandauLifshitz dynamic equations of motion with suitable damping $[7-8]$. The models based on the S-W particles are usually treated as realistic, physical models and have been widely used in the area of magnetic recording [9-11]. Beside the prediction of the complex spin configurations, $\mathrm{dy}$ namics can also give the transient picture that is very important in a high-speed switching recording [12].

Recently, another mathematical hysteresis model, i.e., Preisach model, was susscefully extended to a vector

Manuscript received July 10, 1995.

Jyh-Shinn Yang, e-mail: b0071@ntou66.ntou.edu.tw, fax: 886-24620724; Ching-Ray Chang, e-mail: crchang $@$ phys.ntu.edu.tw, fax: 886-2-3639984; Ivo Klik, fax: 886-2-3639984.

This work was supported in part by National Science Council, ROC, under Grant No. NSC-84-2112-MO02-038. model and it represents a viable alternative to the physically intuitive S-W model [13]. Though it is a pure mathematical, phenomenological model, vector Preisach model in certain way successfully reproduce the $\mathrm{S}-\mathrm{W}$ features at least phenomologically. One of the advantages for the vector Preisach model is quite efficient from a computational point of view because of avoiding the tedious integration procedures [13]. However, the drawback of the vector Preisach model is that they are not ab initio calculations and it is very easy to miss physical interpretation of the results.

To obtain a compromise between computation time and physical reality is a continuous interest in this field, and many researchers have contributed to the improvement of the algorithms [14-16]. The most time-comsumption calculations based on the S-W model are the self-consistent iterations from interactions [1]. Dipole-dipole interactions between particles are usually treated within a microscopic approach or within a phenomenological mean-field model. The microscopic computation requires, as a rule, too much computing power [3]. The mean-field theory allows the simple coherent rotation model to be extended to a interacting system and provides a compromise between accuracy and convenience. For a mean-field model, numerical calculations of the hysteresis loop are usually carried out iteratively, iterating from the loop of assembly of noninteracting particles [1]. For a time-dependent applied field $\vec{H}_{a}(t)$, the effective field is iterated initially with $\vec{H}_{e}^{(0)}(t)=$ $\vec{H}_{a}(t)+\alpha\langle\vec{M}(t)\rangle_{\alpha=0}^{(0)}$ and then self-consistently derives the $i$-th iteration result, the $\langle\vec{M}(t)\rangle_{\alpha}^{(i)}$. However, this algorithm only yields a steeper magnetization curve at coercivity for $\alpha>0$, while the coercivity is never influenced by the interparticle interaction since $\left\langle\vec{M}(t) \cdot \vec{H}_{a}\right\rangle_{\alpha=0}=0$ for $H_{a}(t)=H_{c}$. Therefore, the well-known linear relationship between coercivity and packing density can not be properly demonstrated in an interacting system. Here we present a causality algorithm to keep track of all history of interacting magnetic systems since saturation. Moreover, it reflects the true hysteresis of the interacting system under ambient temperature. The coercivity indeed shifts linearly with increasing coupling strength as observed experimentally. Another benefit from this causality algorithm is that it is based on a realistic model and, at the same time, is also very efficient from computational point 
of view.

\section{THE MODEL}

A classical model for the magnetic reversal is that of uniform rotation against a uniaxial anisotropy [5]. We consider an array of identical single-domain uniaxial particles whose magnetization $\vec{M}$ reverses by coherent rotation. Each particle has energy $E=K V \sin ^{2} \theta-V \vec{M} \cdot \vec{H}_{e}$, where $V$ is the (activation) volume and $K$ the anisotropy constant. The effective magnetic field $\vec{H}_{e}$ includes both the applied field and the interaction field from other particles, $\theta$ is the angle spanned by the particle's easy axis and its magnetization $\vec{M},|\vec{M}|=M_{s}$ by assumption, and $M_{s}$ is the saturation magnetization (Fig. 1).

Interparticle interactions may either be taken into account via a microscopic approach or via the phenomenological mean-field approximation. The microscopic computation requires, as a rule, too much computing power. We here adopt the mean-field approximation in which a representative particle of the array is acted upon by the field $\vec{H}_{e}=\vec{H}_{a}+\alpha\langle\vec{M}\rangle$, where $\vec{H}_{a}$ is the applied magnetic field, $\langle\vec{M}\rangle$ is the instantaneous mean magnetization of the array and $\alpha$ is a phenomenological coupling constant. Calculations of a hysteresis loop within this mean-field model have been reported previously for Stoner-Wohlfarth particles [1]. However, the previous algorithm only treated selfconsistently the noninteracting array and it lost memory of the interacting system itself. The lost-memory effects become more serious at higher temperatures. To include the influence of temperature, we treat an array of many identical particles as having two levels which are occupied with probabilities $n_{1}$ and $n_{2}=1-n_{1}$, respectively. Its evolution is given by the master equation

$$
\dot{n}_{1}=-\kappa_{12} n_{1}+\kappa_{21} n_{2}=-\dot{n}_{2}
$$

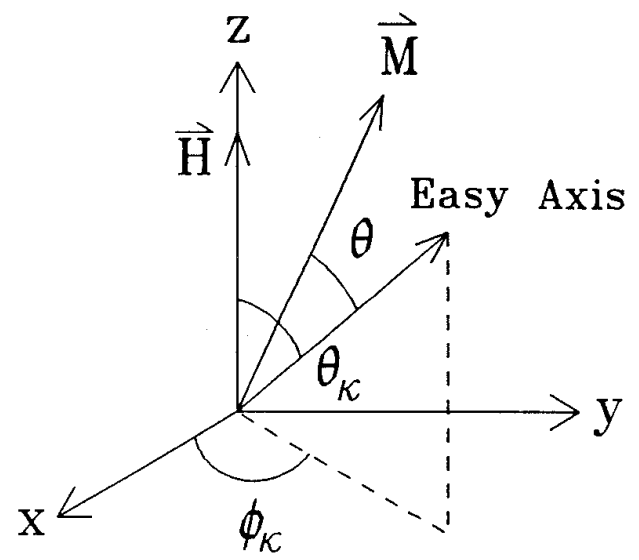

Fig. 1. Schematic diagram for a uniaxial ferromagnetic particle. Easy-axis orientation is defined by the spherical coordinates $\theta_{k}$ and $\phi_{k} . \theta$ is the angle between the magnetization and the easy axis. where $\dot{n}_{i}=d n_{i} / d t$ and $\kappa_{i j}=\kappa_{i \rightarrow j}=f_{0} \exp \left(-Q_{i} / k_{B} T\right)$ is the rate of thermally activated transitions from the $i$-th to the $j$-th level. For the prefactor we choose the value $f_{0}=$ $e^{25} \mathrm{~Hz}, k_{B}$ is the Boltzman constant, $T$ is temperature and $Q_{i}$ is the barrier height to be overcome on departure from the $i$-th well. We shall assume that the applied field driving the hysteresis loop is in the $z$-direction, $\vec{H}_{a}=$ $[0,0, H(t)], H(t)=H_{0} \cos 2 \pi f t$, where $f$ is the sweep rate and the amplitude $H_{0}$ is greater than the nucleation field of the system. In calculating the hysteresis loop we take the amplitude $H_{0}$ sufficiently large for all particles within the array to have initially only one local minimum, i.e., we solve (1) with the initial condition $n_{1}\left[H(t=0) \mid \theta_{k}, \phi_{k}\right]=1$ [17].

The mean-field approximation is implemented as follows: For any easy-axis orientation, only one local energy minimum exists at $t=0$ and we use the easily calculated mean magnetization $\langle\vec{M}(0)\rangle$ to approximate the mean field an instant $d t$ later, setting $\vec{H}_{e}(t)=\vec{H}_{a}(t)+$ $\alpha\langle\vec{M}(t-d t)\rangle$. The updated value of the mean magnetization is then used to approximate the mean field at $t=2 d t$ etc. (Fig. 2). The theory is thus not entirely self-consistent since the response to the coupling field is alway delayed by the integration step $d t$ of the differential equation (1). However, hysteresis really is a memory effect, in particular where thermal relaxation is concerned, and the present approach is certainly preferable to the iterative procedure proposed by Atherton and Beattie for the causality problem treated here $[1]$.

\section{RESULTS AND DISCUSSIONS}

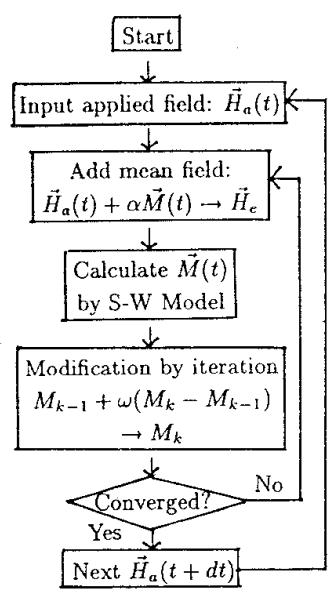

(a)

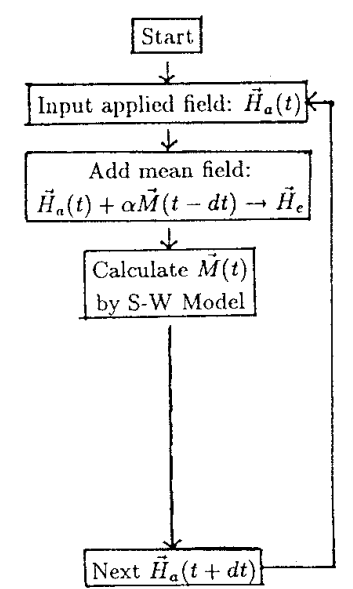

(b)
Fig. 2. Comparison of flowcharts for the self-consistent algorithm (a) and the causality algorithm (b). 
The previously discussed self-consistent algorithm [1] maintains only the history of the noninteracting system, disregarding the evolution due to the interactions while the present causality approach keeps track of all history since saturation. It reflects the true hysteresis of the interacting system, for a two-level inetaracting system, and coercivity shifts outwards with increasing positive interaction strength (Fig. 3). In comparison with the selfconsistent algorithm, the present algorithm does not only produce the correct physical behavior but also saves a lot of computation time. Moreover, the algorithm is not only applicable at zero temperature, e.g., S-W model, it is also useful for magnetically interacting systems with finite temperature [17]. As shown in Fig. 4, the stronger the coupling strength, the better performance the present algorithm does. The CPU time becomes $1 / 9$ of that for the self-consistent algorithm at the strongly interacting systems. Therefore, though not entirely self-consistent, the casulity algorithm satisfies the requirement of causality and is physically more realistic. The mean-field theory discussed here has recently been shown to be applicable to interacting systems dominated by ferromagnetic ordering [18]. Such systems, namely, have essentially only two levels (states) in which all particles are, as much as possible, aligned in parallel resp. antiparallel direction to the applied field. Equation (1) should therefore be interpreted as the probability of finding the entire system in one of its two possible metastable configurations and in the limit $\alpha \rightarrow 0$, one then recoveres the single-particle probability. Systems dominated by antiferromagnetic ordering display additional metastable states which are partially or fully demagnetized. Equation (1), which refers to the probabi-

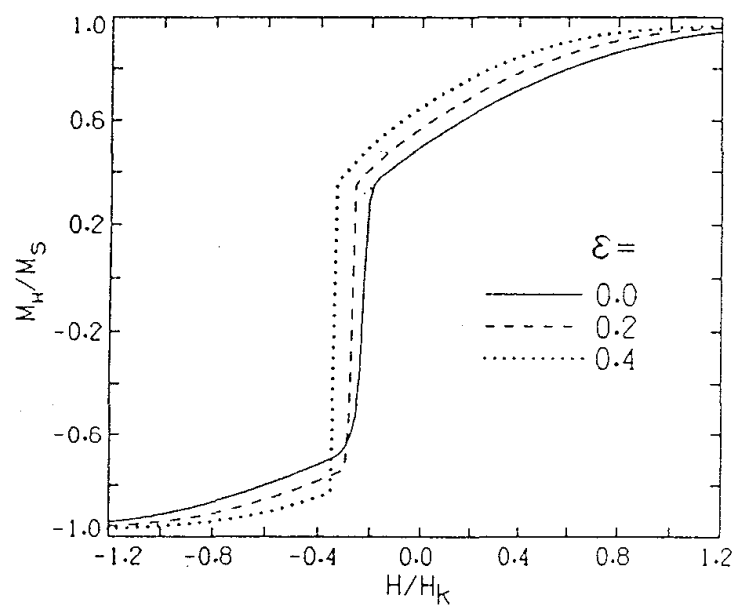

Fig. 3. Hysteresis curves of the uniformly distributed easy axes system with different reduced coupling strength $\mathcal{E}$. Here $\mathcal{E}=\alpha M_{s} / H_{k}$, $H_{k}=2 K / M_{s}, K V / k_{B} T=50$, and $f=0.1 \mathrm{~Hz}$. lity of the system being fully magnetized in one of two possible directions is then obviously inapplicable since in this case there exists a nonzero probability that the system finds itself in one of the additional, demagnetized states. However, a causal mean-field theory based on more than two levels is certainly possible and is currently under development by the authors.

In summary, we have presented a new algorithm for the hysteresis of interacting systems. This algorithm not only keeps the history from the very beginning but also saves a lot of computation time, especially for strongly interacting systems. The interacting system calculated from this mean-field algorithm can properly demonstrate the realistic, physical behaviors of the coercivity with the suitably chosen $\alpha$. Although the model is not strictly correct from the first-principles point of view, it still provides a workably phenomenological model of the hysteresis. This casuality algorithm not only saves a lot of computation time, as a mathematical, vectorial Preisach model does, but also correctly reflects the physical picture.

\section{REFERENCES}

[1] D. Atherton and J. R. Beattie," A mean field Stoner-Wohlfarth hysteresis model," IEEE Trans. Magn., vol. 26, no. 6, pp. 30593063, 1990.

[2] I. D. Mayergoyz, "Vector Preisach hysteresis models," J. Appl. Phys., vol.63, no. 8, pp. 2995-3000, 1988.

[3] J. G. Zhu and H. N. Bertram, "Micromagnetic studies of thin metallic films," J. Appl. Phys., vol.63, pp. 3248-3253, 1988.

[4] Ching-Ray Chang and Jyh-Shinn Yang, "Detection of anisotropy in the reversible transverse susceptibility," Appl. Phys. lett., vol.65, no. 4, pp. 496-498, 1994.

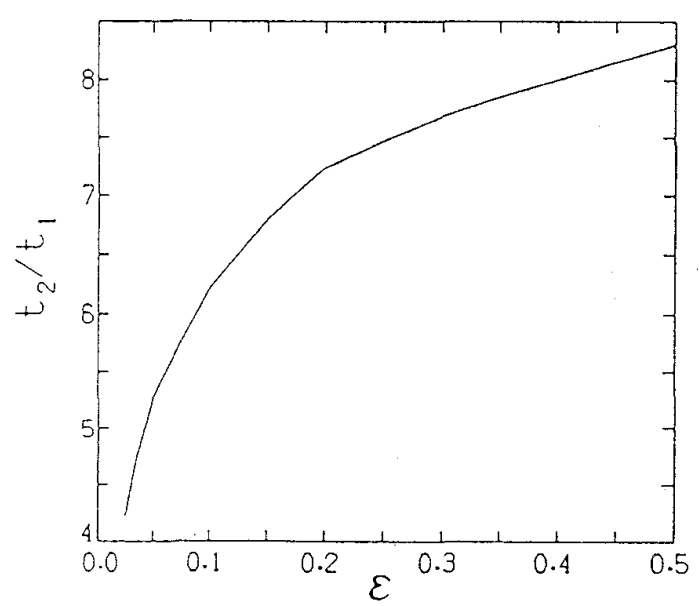

Fig. 4. Ratio of $t_{2}$ to $t_{1} v s$ reduced coupling strength $\mathcal{E}$. $t_{2}$ and $t_{1}$ are the average $\mathrm{CPU}$ time in computating the hysteresis curves according to the self-consistent and present algorithms, respectively. 
[5] E. C. Stoner and E. P. Wohlfarth, "Mechanism of magnetic hysteresis in heterogenous alloys," Phil. Trans. Royal Soc. London, vol. A240, pp. 599-642, 1948.

[6] Ching-Ray Chang and D. R. Fredkin, "The dynamic vectorial model for assemblies of Acicular Particles," IEEE Trans. Magn., vol. MAG-22, no. 5, pp. 391-393, 1986.

[7] H. N. Bertram and J.-G. Zhu, "Fundamental magnetization processes in thin-film recording media," in Solid State Physics, vol. 46, New York: Academic, 1992, pp. 271-371.

[8] Y. Nakatani, Y. Uesaka, and N. Hayashi, "Direct Solution of the Lauau-Lifshitz-Gilbert equation for micromagnetics," Jpn J. Appl. Phys., vo. 28, no. 12, pp.2485-2507, 1989.

[9] R. I. Potter and I. Beardsley, "Self-consistent computer calculations for perpendicular magnetic recording," IEEE Trans. Magn., vol. MAG-16, no. 5, pp.967-972, 1980.

[10] I. Beardsley, "Modeling the recording process," IEEE Trans. Magn., vol. 22, no. 5, pp. 454-459, 1986.

[11] T. R. Koehler, "A computationally fast, two-dimensional vector hysteresis model," J. Appl. Phys., vol.61, pp. 1568, 1987.
[12] E. D. Dahlberg and J.-G. Zhu, "Micromagnetic microscopy and modeling," Physics Today, April, pp.34-42, 1995.

[13] I. D. Mayergoyz, Mathematical models of hysteresis, New York: Springer-Verlag, 1991, pp. 141-194.

[14] G. F. Hughes, "Magnetization reversal in cobalt-phosphorus films," J. Appl. Phys., vol. 54, no.9, pp.5306-5313, 1983.

[15] T. R. Kohler, "Mapping procedure to match hysteresis loops from models to experimental results," IEEE Trans. Magn., vol. 22 , no. 5, pp. 1056-7, 1986.

[16] A. L. Ribeiro, "Magnetic hysteresis model for magnetic recording including spatial fluctuations of interaction fields," J. Appl. Phys., vol. 69, no. 8, pp. 4841-4843, 1991.

[17] Jyh-Shinn Yang, Ching-Ray Chang, and Ivo Klik, "Thermomagnetics of reversible susceptibility," Phys. Rev. B, in press, 1995.

[18] Ivo Klik and Ching-Ray Chang, "Thermal relaxation in arrays of coupled ferromagnetic particles," Phys. Rev. B, in press, 1995. 